Revue internationale P.M.E.

Économie et gestion de la petite et moyenne entreprise

\title{
Pratiques innovatrices des PME rhône-alpines : une approche par la diversité des sources de l'innovation technologique
}

\section{Brigitte Guedj et Fabienne Picard}

Volume 7, numéro 3-4, 1994

URI : https://id.erudit.org/iderudit/1008423ar

DOI : https://doi.org/10.7202/1008423ar

Aller au sommaire du numéro

Éditeur(s)

Presses de l'Université du Québec

ISSN

0776-5436 (imprimé)

1918-9699 (numérique)

Découvrir la revue

Citer cet article

Guedj, B. \& Picard, F. (1994). Pratiques innovatrices des PME rhône-alpines : une approche par la diversité des sources de l'innovation technologique. Revue internationale P.M.E., 7(3-4), 41-64. https://doi.org/10.7202/1008423ar
Résumé de l'article

Cet article vise à décrire le comportement des petites et moyennes entre- prises (PME de 20 à 499 salariés) rhône-alpines en matière de création et d'acquisition des connaissances technoscientifiques nécessaires à la réalisation des innovations technologiques. Pour mener à bien cette analyse, nous avons utilisé les résultats d'une régionalisation de l'enquête innovation du SESSI (1990). On montre dans un premier temps que le critère de la taille des entreprises innovantes permet d'identifier des comportements types qui opposent les PME aux grandes entreprises. Mais cette homogénéité cache en réalité une diversité comportementale des PME innovatrices mise en évidence lors de l'analyse sous-sectorielle. On identifiera notamment trois grands types de sous-secteurs se caractérisant par le développement de pratiques d'acquisition de connaissances technologiques externes distinctes. 


\title{
Pratiques innovatrices des PME rhône-alpines: une approche par la diversité des sources de l'innovation technologique
}

Brigitte GUEDJ*

Fabienne PICARD**

Université Lumière Lyon 2

\section{MOTS CLÉS}

\section{Enquêtes innovation - Innovation technologique \\ Pratiques innovatives - Recherche et développement \\ Réseaux technoscientifiques - Sources d'innovation}

\begin{abstract}
RÉSUMÉ
Cet article vise à décrire le comportement des petites et moyennes entreprises (PME de 20 à 499 salariés) rhône-alpines en matière de création et d'acquisition des connaissances technoscientifiques nécessaires à la réalisation des innovations technologiques. Pour mener à bien cette analyse, nous avons utilisé les résultats d'une régionalisation de l'enquête innovation du
\end{abstract}

* Brigitte Guedj prépare actuellement une thèse de doctorat en Sciences économiques à Lyon 2 sur le thème de l'innovation. Elle a participé à la régionalisation de l'enquête innovation du SESSI dans le cadre d'un rapport de stage de DESS «Évaluation et maîtrise de l'innovation et de la technologie» effectué à la Direction régionale de l'INSEE Rhône-Alpes. Adresse : Université Lumière Lyon 2, Faculté de sciences économiques et de gestion, 16, Quai Claude-Bernard, 69365 Lyon, Cédex 07, France.

** Fabienne Picard est allocataire de recherche au Laboratoire d'économie et statistique appliquées. Elle prépare une thèse de doctorat en Sciences économiques à Lyon 2 concernant les problèmes de rendements de la recherche industrielle. Adresse: Université Lumière Lyon 2, Faculté de sciences économiques et de gestion, 16, Quai Claude-Bernard, 69365 Lyon, Cédex 07, France. 
SESSI (1990). On montre dans un premier temps que le critère de la taille des entreprises innovantes permet d'identifier des comportements types qui opposent les PME aux grandes entreprises. Mais cette homogénéité cache en réalité une diversité comportementale des PME innovatrices mise en évidence lors de l'analyse sous-sectorielle. On identifiera notamment trois grands types de sous-secteurs se caractérisant par le développement de pratiques d'acquisition de connaissances technologiques externes distinctes.

\section{ABSTRACT}

The purpose of the paper is to describe the small and medium sized firms' (SME) sources of technological innovation. To this end we use a "SESSI" regionalized database (1990). Results are presented of an empirical study of the Rhône-Alpes region covering a sample of 2714 innovatives firms. The findings indicate that SMEs' strategies of technological resources acquisition are different from those of big enterprises. But a sectoral analysis of small and medium sized firms shows that there are three kinds of technology-oriented external relationships.

\section{RESUMEN}

Objectivo de este artículo es de describur el comportemento de las pequeñas y medias empresas, tratándose de fuentes de innovaciónes tecnológicas. Per eso, hemos utilisado una regionalización de la encuesta "innovación " del SESSI (1990). Primero, podemor ver que la estrategia de adquisicion de les fuentes de innovaciónes tecnológicas es distinta de esta de las empresas mayores. Pero, el analisis sectoral de las pequeñas y medias empresas demonstra que tre estrategias existan para la estrategia de adquisicion de las fuentes de innovaciónes.

\section{Introduction}

L'objet de cet article est de rendre compte du comportement en matière d'innovation technologique des petites et moyennes entreprises (PME, de 20 à 499 salariés) de la région Rhône-Alpes. Il s'agit, plus précisément, de caractériser la genèse de leurs innovations par l'identification des modalités d'acquisition des ressources scientifiques et technologiques. Cœur de la compétitivité industrielle, l'innovation contribue à la survie des firmes dans un environnement économiquement instable. Si l'on octroie désormais à l'innovation technologique le statut de variable stratégique, force est de constater que tel n'a pas toujours été le cas. En effet, l'innovation a longtemps été considérée comme une «boîte noire», «nos connaissances statistiques et nos indicateurs se contentaient d'en saisir les "inputs" (les ressources affectées à la R-D par exemple) et les outputs (brevets, exportations ou parts des marchés) » (Barré, 1991). Or, l'innovation requiert une statistique plus complexe, notamment, en ce qui concerne ses inputs. Diverses analyses ont attesté 
du simplisme d'une conceptualisation d'un processus de création de technologies, elles-mêmes d'une complexité croissante, qui confère aux seules ressources internes de $\mathrm{R}-\mathrm{D}$ cette prérogative innovative (conformément à la représentation linéaire de ce processus).

Une idée maîtresse veut qu'il existe manifestement un hiatus entre la reconnaissance de la complexité des connaissances, des compétences requises et l'acceptation de l'unicité de l'origine des ressources mobilisées. La recherche et développement (désormais $R-D$ ) dans sa définition actuelle n'apparaît pas comme indicateur satisfaisant pour l'évaluation des inputs innovatifs, particulièrement en ce qui concerne les PME (Kleinknecht, 1987). Le processus d'innovation est un phénomène mieux approché par une représentation conceptuelle interactive (Kline, 1985; Kline et Rosenberg, 1986) où la multiplicité des chemins par lesquels peut naître une innovation renvoie à la diversité des sources de celle-ci. Cette cristallisation de sources hétérogènes autour de l'innovation peut s'expliquer par la complexité croissante et la diversité des connaissances nécessaires à la maîtrise de ce processus. En effet, "the "solution" of technological problems involves the use of information drawn from previous experience and formal knowledge [...]; however, it also involves specific and uncodified capabilities on the part of "inventors"1" (Dosi, 1988). La création de connaissances ou technologies nouvelles est donc un processus cognitif intrinsèquement complexe. Elle suppose non seulement la mobilisation de compétences, de bases de connaissances scientifiques et technologiques d'origines diverses, construites à partir de l'agrégation de connaissances anciennes et nouvelles, mais également leur recontextualisation dans une logique productive et commerciale spécifique à chaque entreprise. Par ailleurs, ce processus est très coûteux, notamment pour les PME qui disposent généralement de fonds propres en quantités limitées.

Compte tenu des investissements substantiels qu'implique l'innovation, l'hypothèse pourrait être avancée que les PME recourraient essentiellement à des sources externes pour nourrir leur processus d'innovation. Nous verrons qu'en réalité ce n'est pas en ces termes que se pose le problème de la création des innovations technologiques. Il repose davantage sur une complémentarité entre sources d'origines diverses que sur une substituabilité entre celles-ci. On tient là une extension à l'ensemble des sources de l'innovation de l'hypothèse émise par Cohen et Levinthal (1989) concernant la complémentarité entre des activités de recherche interne et externe.

1. «La solution des problèmes technologiques suppose le recours à l'information provenant de l'expérience antérieure et de la connaissance [...]; cependant, elle requiert aussi des capacités spécifiques et non codifiées de la part des inventeurs» - Traduction libre. 
Cet article propose un descriptif des pratiques d'innovation technologiques développées par les PME rhône-alpines à partir des tableaux de résultats régionalisés de l'enquête innovation du SESSI (1990). Une première section sollicite la littérature contemporaine en vue d'identifier un cadre analytique pertinent, en l'occurrence celui des réseaux d'innovation. On décrit ensuite la genèse de l'innovation technologique en fonction de la taille de l'entreprise, tandis qu'une troisième section vise à repérer l'existence de logiques sous-sectorielles dans l'utilisation et l'association des différentes sources.

\section{Sources de l'innovation technologique et réseaux technoscientifiques}

L'analyse économique du changement technique et de l'innovation se structure autour de deux approches méthodologiques distinctes, plus complémentaires qu'antagonistes, en matière d'analyse des sources de l'innovation: l'analyse intersectorielle du changement technologique et l'analyse des réseaux technoscientifiques. L'objet de cette section est de montrer la pertinence de cette dernière. En effet, si l'analyse intersectorielle du changement technique met en relief la dynamique innovatrice globale en localisant l'origine intra- ou extrasectorielle de l'innovation ${ }^{2}, 1$ 'approche en termes de réseaux technoscientifiques permet de cerner les interactions entre les différentes sources d'innovation telles qu'elles peuvent se créer entre et au sein des unités productives.

Au niveau de la firme, une partition quasi analogue à celle qui oppose l'origine intrasectorielle à la provenance extrasectorielle de l'innovation s'impose avec une même pertinence, sans pour autant se limiter au champ de l'appartenance sectorielle. L'hypothèse sous-jacente à cette approche, distinguant les sources de l'innovation internes et externes à la firme, veut que l'innovation soit un processus qui résulte bien souvent de l'interaction entre ces différentes sources, porteuses de compétences et d'informations diverses. La firme innovante devient le lieu de rencontre de deux formes d'apprentissage

2. La localisation de l'origine sectorielle d'une innovation ou d'un changement technique a pu être établie selon diverses méthodologies qui reposent sur l'évaluation marchande ou non marchande des flux technologiques entre secteurs (De Bresson et Amesse, 1991). Les biens de consommation intermédiaire constituent un premier vecteur de suivi des transferts de connaissances techniques entre les industries (Terleckyj, 1974 ; Momigliani et Siniscalco, 1984). Une deuxième méthode s'appuie sur la construction de matrices à partir des flux de brevets (Scherer, 1982) ou, plus récemment, des innovations technologiques (Robson et al., 1988). 
technologique, interne et externe (Dodgson, 1991), chacune insuffisante en soi, mais nécessaire à l'autre (Le Bas, 1993). L'objectif de cette démarche n'est pas d'établir une hiérarchisation entre les différents types de sources, mais d'identifier les sources qui, contribuant à la création et à l'acquisition des innovations technologiques, participent à la dynamique innovative des firmes industrielles.

Notre recherche s'inspire des analyses en termes de réseaux, ceux-ci étant conçus non comme une forme d'organisation intermédiaire entre marché et hiérarchie (Thorelli, 1986; Powell, 1990; Bidault, 1993), mais comme un ensemble de relations technoscientifiques entre des acteurs et des techniques (Callon, 1991). Le concept de réseau technoscientifique, défini "comme un ensemble coordonné d'acteurs hétérogènes [...] qui participent collectivement à la conception, à l'élaboration, à la production et à la distribution-diffusion des procédés de production de biens et de services, dont certains donnent lieu à une transaction marchande » (Callon, 1991), s'avère un instrument d'analyse pertinent pour structurer les réflexions développées autour de la relation entreprise-environnement, en intégrant la complexité et la diversité des ressources technologiques (Rapport du commissariat général du Plan, 1993).

Néanmoins, la multiformité et le caractère évolutif du réseau en rendent la connaissance exhaustive difficile et requièrent une catégorisation de ses composants. Celle-ci peut être menée selon trois grandes perspectives. La première identifie la nature institutionnelle des organismes contactés par une firme, la deuxième s'intéresse à la nature des vecteurs d'information entre les acteurs, la troisième s'attache à distinguer les types de comportements en matière d'élaboration de relations réticulaires.

L'approche des réseaux en fonction de l'appartenance institutionnelle des acteurs constitutifs ${ }^{3}$ se révèle particulièrement utile lors de l'étude des politiques publiques d'aide au développement de l'innovation. Ces formes institutionnelles entre lesquelles se développent des relations technoscientifiques conditionnent dans une certaine mesure la nature du vecteur de l'échange (de l'intermédiaire au sens de Callon, 1991) et renvoient en définitive à une analyse morphologique des réseau ${ }^{4}$. Le repérage de ces vecteurs

3. Parmi ces acteurs, on distingue des organismes publics ou privés de recherche, des universités, des organismes de transfert de technologie, d'autres entreprises appartenant ou non à la même industrie, des clients, des fournisseurs, etc.

4. On retrouve ici regroupés l'ensemble des éléments isolés dans les différents travaux d'analyse intersectorielle qui identifient la source de l'innovation (R-D de différents types, brevets, licences, intrants technologiques...). 
d'information permet d'identifier l'existence d'un réseau. L'accès aux ressources externes peut revêtir principalement deux aspects. L'un, formel et marchand, permet à la firme de s'approprier des technologies par l'achat de biens intermédiaires, de brevets, de licences, d'entreprises (dans une perspective éducative), par la contractualisation de la recherche, ou l'embauche de personnels qualifiés (Dussauge et Ramanantsoa, 1987). On parlera dans ce cas de liaisons formelles. Quant aux autres moyens de diffusion et de captation des connaissances technologiques non objectivées dans des biens ou contrats, ils se présentent sous des aspects non formalisés, et parfois même non marchands (Kelley et Brooks, 1991). Ces liaisons informelles regroupent l'ensemble des flux informationnels (médias, brochures, salons, conférences, séminaires, relations personnelles), ainsi que le dialogue avec des firmes clientes ou des fournisseurs.

Enfin, les relations qu'une firme tisse avec son environnement s'architecturent autour de deux comportements types à l'égard des ressources fournies par l'extérieur: l'un, passif de simple réception ; l'autre, actif de création (selon les termes de Kelley et Brooks, 1991). Ces comportements typiques aboutissent à une double structuration en termes de réseaux. Aux réseaux de liaisons passives, caractérisés par un comportement d'acquisition de connaissances, de compétences externes requérant un faible engagement de l'entreprise à l'égard de la création de ces ressources externes (la firme reste un réceptacle qui se limite à la stricte acquisition des technologies dont elle a besoin), on oppose un réseau créatif, participatif constitué par des liaisons actives, dans lequel s'insère la firme en vue de contribuer, avec d'autres agents, à la création d'une innovation. Cette distinction renvoie aux acceptions respectivement extensives et strictes des réseaux (infra).

Il n'existe pas, à notre connaissance, d'études systématiques (autres que celles portant sur de petits échantillons) ayant pour objet l'analyse simultanée de ces différentes caractéristiques. Or, l'enquête innovation du SESSI, sur laquelle nous nous appuyons, permet de rendre compte de pratiques innovatives des PME, non nécessairement réductibles aux activités organisées en interne par la firme. Les réseaux d'acquisition des ressources technologiques externes des PME sont appréhendés à partir des résultats régionalisés de cette enquête. Nous traiterons des diverses pratiques innovatrices en fonction de la taille des firmes, d'une part, et de leur appartenance sectorielle, d'autre part. 


\section{Genèse de l'innovation technologique et taille des firmes}

\subsection{Méthodologie de l'enquête innovation du SESSI et caractérisation de l'échantillon étudié}

Pour réaliser cette étude, nous avons analysé les résultats issus de l'enquête innovation mise au point par le SESSI (Service statistiques du ministère de l'Industrie) et jointe à l'Enquête annuelle d'Entreprises de 1990. Adressé à l'ensemble des entreprises industrielles françaises de plus de vingt salariés ${ }^{5}$, ce questionnaire EAE-SESSI visait à améliorer la connaissance de l'activité innovative des firmes industrielles au cours des cinq dernières années. À cette occasion, des informations sur la nature des innovations (technologiques [produit, procédé] commerciales et organisationnelles), leurs stimuli (demande ou offre de nouvelles technologies), leurs incidences sur le chiffre d'affaires, les exportations et la place des différentes sources technologiques dans la genèse de l'innovation ont été recueillies (François, 1991). Les résultats nationaux et régionaux établis à partir de l'ensemble de cette enquête ont fait l'objet de plusieurs publications ${ }^{6}$.

L'important ici n'est pas de décrire l'activité innovatrice suivant ses diverses modalités, mais de focaliser sur les pratiques de création ou d'acquisition des connaissances scientifiques et technologiques à l'origine de l'innovation technologique. Cette approche sera conduite au niveau régional. Dans cette optique, une régionalisation ${ }^{7}$ des résultats de l'enquête innovation du SESSI a été menée par l'INSEE Rhône-Alpes. Elle a permis d'isoler quelque 4115 entreprises dont $66 \%$ se sont déclarées innovantes en produits ou procédés ${ }^{8}$. Conformément aux approches en termes de réseaux, les sources

5. Une enquête similaire a été réalisée auprès des très petites entreprises industrielles (TPEI). Les résultats sont présentés dans de Barry et Savoye, 1993.

6. Auzeby et François, 1992 ; Bernard et Torre, 1994, pour une présentation des résultats au niveau national, une première mise en perspective spatiale proposée dans La Lettre de l'OST (1991), tandis que des études exclusivement régionales tendent à se développer également (Jafra et Zins, 1993; Guedj, 1993).

7. Une entreprise sera considérée comme appartenant à la région Rhône-Alpes dès lors qu'elle détient plus de $50 \%$ de son effectif ou plus de 100 salariés en RhôneAlpes.

8. N'ont été sélectionnées que les entreprises ayant déclaré avoir introduit au moins une innovation en matière de produits ou de procédés, au cours des cinq dernières années. Parmi ces firmes, certaines peuvent avoir également innové d'un point de vue organisationnel ou commercial, mais ce sont des informations dont nous ne tenons pas compte. De même, la qualité et le nombre des innovations ne sont pas pris en considération. 
d'innovation répertoriées (au nombre de huit) peuvent être classées selon trois sous-catégories (que l'on retrouve dans toutes les études citées en note 6).

Les sources dites «internes » regroupent l'activité de R-D permanente et organisée dans l'entreprise (au moins un scientifique, chercheur ou ingénieur annuel en équivalent temps plein), l'activité d'étude technique et de méthode interne à l'entreprise - y compris de la R-D non permanente - et les brevets dont l'entreprise est titulaire. Les sources dites «externes» se décomposent en R-D interne au groupe auquel appartient l'entreprise, acquisition de R-D à l'extérieur (auprès d'organismes publics, professionnels ou privés de recherche, clients...), acquisition à l'extérieur de l'entreprise de droits et de licences d'industrialisation relatifs à des brevets, des inventions et des savoir-faire techniques ${ }^{9}$. Enfin, sont également pris en compte les intrants technologiques tels que l'utilisation novatrice de biens d'investissements (installation, processus, matériels), ainsi que la mise en œuvre de matériaux et composants nouveaux pour l'entreprise. L'originalité de cette enquête réside dans la reconnaissance de la diversité des sources d'innovation qui permet de mieux cerner leur contribution relative à la dynamique innovative des firmes ${ }^{10}$.

\subsection{Quelques résultats globaux quant à la genèse de l'innovation technologique en Rhône-Alpes selon la taille des firmes}

L'enquête innovation du SESSI (1990) offre un premier descriptif comportemental des entreprises en matière d'acquisition d'innovations technologiques. À partir de ces résultats, une première étude peut être menée afin de mettre en relief l'impact de la taille de l'entreprise sur les sources d'innovation qu'elle utilise. Il s'agit de déterminer dans quelle mesure le recours à l'une ou l'autre des sources d'innovation est lié à la taille de l'entreprise innovante et ainsi de repérer l'existence d'une spécificité comportementale des PME comparativement aux grandes entreprises.

L'étude des sources d'innovation repose sur le postulat que les entreprises innovent. Aussi, un préalable consiste en une estimation de l'importance quantitative de ce phénomène relativement à la taille de la firme.

9. La création de connaissances technologiques dans le cadre d'une coopération intentionnelle ne sera pas étudiée.

10. Soulignons, cependant, que le caractère qualitatif des variables étudiées et le fait qu'une même entreprise peut déclarer avoir utilisé simultanément plusieurs sources, réduisent les traitements statistiques envisageables. 
TABleau 1

Profil des entreprises innovantes de la région Rhône-Alpes

\begin{tabular}{l|c|c|c|c|c}
\cline { 2 - 6 } & $\begin{array}{c}\text { Petites } \\
\text { entreprises } \\
\mathbf{2 0 - 9 9}\end{array}$ & $\begin{array}{c}\text { Moyennes } \\
\text { entreprises } \\
\mathbf{1 0 0 - 4 9 9}\end{array}$ & $\begin{array}{c}\text { PME } \\
\mathbf{2 0 - 4 9 9}\end{array}$ & $\begin{array}{c}\text { Grandes } \\
\text { entreprises } \\
\mathbf{5 0 0} \text { et + }\end{array}$ & Total \\
\hline Nombre d'entreprises & 3246 & 639 & 3885 & 230 & 4115 \\
\hline $\begin{array}{c}\text { Répartition des firmes } \\
\text { par taille }\end{array}$ & $79 \%$ & $15,5 \%$ & $94,5 \%$ & $5,5 \%$ & $100 \%$ \\
\hline $\begin{array}{c}\text { Firmes déclarant } \\
\text { avoir innové }\end{array}$ & $61 \%$ & $80 \%$ & $65 \%$ & $92 \%$ & $66 \%$ \\
\hline
\end{tabular}

Sources : SESSI (1990), INSEE Rhône-Alpes (1993).

On constate que sur les l'ensemble des entreprises interrogées (toutes tailles confondues), $66 \%$ déclarent avoir innové au cours des cinq dernières années (contre $60 \%$ au niveau national). La propension à se déclarer innovante est d'autant plus grande que croît la taille de l'entreprise (en termes d'effectifs salariés). En effet, alors que $61 \%$ des petites entreprises se sont déclarées innovantes, ce pourcentage passe à $80 \%$ pour les moyennes et à $92 \%$ pour les grandes ${ }^{11}$. Cela étant, une question demeure : quelle est la genèse de l'innovation technologique, c'est-à-dire la nature des vecteurs utilisés par les entreprises pour innover?

Une analyse des données nationales de la distribution des sources d'innovation des firmes innovantes a été menée par J. Bernard et A. Torre (1994). Étudiant les sources d'innovation utilisées de manière intensive par les entreprises en fonction de leur taille, ils montrent que si les grandes entreprises recourent plus à la R-D interne, les PME privilégient quant à elles l'activité d'étude technique. Concernant les sources d'innovation externes à l'entreprise, la taille de cette dernière n'apparaît pas comme un élément déterminant de l'acquisition de droits et de licences... ou de R-D externe. En revanche, l'accès à la R-D interne au groupe est d'autant plus aisé que la taille de la firme est importante. Ces résultats nationaux sont confirmés au niveau régional. Cependant, ils ne nous semblent pas des plus révélateurs, car ne sont prises en compte que les déclarations d'utilisation intensive des sources d'innovation. La pertinence de ce critère reste aléatoire et soumise à la subjectivité des réponses dans la mesure où la perception de l'importance d'une source d'innovation peut varier en fonction de la taille de l'entreprise et de

11. La qualité des innovations introduites respectivement par les entreprises de différentes tailles n'est pas prise en compte. 
son secteur d'activité. Aussi, l'analyse que nous développons s'appuie-t-elle sur un regroupement binaire des réponses positives, d'une part, et négatives, de l'autre ${ }^{12}$. Les résultats obtenus s'avèrent significativement différents des précédents, mais trouvent là encore confirmation au niveau national (Auzeby et François, 1992).

TABLEAU 2

Répartition des sources de l'innovation technologique par taille de firmes (Rhône-Alpes)

\begin{tabular}{l|c|c|c|c}
\cline { 2 - 5 } & \multicolumn{4}{c}{ Taille des entreprises } \\
\hline Sources d'innovation déclarées & $\begin{array}{c}\text { PE } \\
\mathbf{2 0 - 9 9} \\
\mathbf{\%}\end{array}$ & $\begin{array}{c}\text { ME } \\
\mathbf{1 0 0 - 4 9 9} \\
\mathbf{\%}\end{array}$ & $\begin{array}{c}\text { PME } \\
\mathbf{2 0 - 4 9 9} \\
\mathbf{\%}\end{array}$ & $\begin{array}{c}\text { GE } \\
\mathbf{5 0 0} \text { et + } \\
\mathbf{\%}\end{array}$ \\
\hline R-D interne & 44 & 64 & 49 & 88,6 \\
\hline Activités d'étude et méthode technique & 74 & 87 & 76,5 & 94 \\
\hline Brevets appartenant à l'entreprise & 23 & 49 & 26,5 & 68 \\
\hline R-D du groupe & 17 & 36 & 20,7 & 70,7 \\
\hline R-D externe & 42 & 54 & 44 & 62 \\
\hline Droits acquis à l'extérieur & 16 & 25 & 18 & 43 \\
\hline Biens d'investissement & 74 & 82 & 75,5 & 87 \\
\hline Composants nouveaux & 58 & 70 & 60,7 & 79,2 \\
\hline
\end{tabular}

Sources : SESSI (1990), INSEE Rhône-Alpes (1993):

Quelle que soit la taille de l'entreprise, la source d'innovation la plus fréquemment déclarée par les 4115 entreprises rhône-alpines est l'activité d'étude technique et de méthode $(76,5 \%$ pour les PME et $94 \%$ pour les grandes entreprises). Toutefois, pour les grandes entreprises, viennent successivement la R-D interne (88,6\%) et les intrants technologiques ( 87 et $79,2 \%$ ), tandis que les PME déclarent utiliser ensuite le plus souvent des intrants technologiques $(75,5$ et $60,7 \%)$, la R-D interne n'étant citée qu'en quatrième position $(49 \%)$. Enfin, dans tous les cas de figure, la source la moins signalée est l'acquisition de droits et de licences (18\% pour les PME et $43 \%$ pour les grandes entreprises). Ces éléments indiquent clairement la prédominance des sources internes et des intrants technologiques comme vecteurs principaux d'innovation et une moindre sollicitation des sources externes.

12. Parmi les réponses positives est introduit un degré d'intensité de contribution de la source à l'innovation: un peu, moyennement ou beaucoup. 
Par ailleurs, on remarque que le pourcentage de sources déclarées augmente avec la taille des firmes. Chaque entreprise pouvant déclarer concomitamment plusieurs sources, on constate que plus les entreprises sont grandes, plus elles ont tendance à recourir à différentes sources. On retrouve certainement là une des forces des entreprises de grande taille: la capacité de démultiplier les sources d'information. Ainsi, non seulement la répartition structurelle des ressources utilisées varie avec la taille de la firme, mais la capacité de combiner des sources d'origines très diverses se présente également comme un élément significatif de différenciation des grandes entreprises et des PME.

Lorsqu'on s'intéresse plus en détail au comportement respectif des petites (20-99) et des moyennes entreprises (100-499), on ne constate pas de disparités significatives. La source d'innovation la plus fréquemment déclarée par les entreprises de petite taille est celle liée aux activités d'études à égalité avec l'utilisation novatrice de biens d'investissements (74\%); viennent ensuite l'acquisition de composants nouveaux (58\%) et en quatrième position la R-D interne (44\%). Pour les entreprises de taille moyenne, on retrouve la même hiérarchisation, mais à des niveaux de pourcentage plus élevé. Finalement, la distinction entre des entreprises de petites ou moyennes tailles n'est pas un facteur discriminant de la structuration des sources de l'innovation technologique des PME.

L'activité de R-D a longtemps été considérée comme la source principale de la création technologique. Alors que la plupart des grandes entreprises innovantes ( $92 \%$ des grandes entreprises) déclarent utiliser de la R-D interne ( $89 \%)$, force est de constater qu'en ce qui concerne les PME l'écart est substantiel entre les firmes qui innovent $(65 \%)$ et celles qui, pour ce faire, utilisent de la R-D interne ( $49 \%)$. Cette comparaison indique très clairement que l'innovation dans les PME, plus que dans les grandes entreprises, ne peut provenir uniquement d'une activité de R-D interne. Le constat s'impose d'une activité de R-D interne n'épuisant pas la question des sources d'innovation des PME. Dès lors, deux perspectives s'offrent aux PME innovantes qui puisent leur innovation ailleurs qu'en R-D interne. La première consiste à développer une activité interne à l'entreprise différente de la recherche stricto sensu telle que la définissent les statisticiens (de Frascati, 1981). Il s'agit en l'occurrence de l'activité d'étude technique et de méthode. Cette activité participe largement au développement des innovations ou améliorations technologiques sans recourir à une recherche scientifique permanente et organisée. Elle est également le lieu d'expression d'une recherche développée ponctuellement par l'entreprise. On constate effectivement son importance comme source d'innovation (tableau 2), puisque c'est la source la plus fréquemment citée, quelle que soit la taille de l'entreprise. La seconde 
solution pour les PME qui ne peuvent effectuer une R-D interne permanente, faute de ressources (humaines, financières ou matérielles) suffisantes ou adéquates, consiste à l'acquérir auprès d'organismes extérieurs. On verra que le choix de l'une ou l'autre de ces deux options est éclairé par l'appartenance sectorielle de l'entreprise (l'existence de centres techniques ou professionnels spécifiques à certains secteurs d'activité favorise l'utilisation d'un certain type de R-D externe).

Cette approche de la genèse de l'innovation révèle que les entreprises, quelle que soit leur taille, mobilisent une panoplie de sources et construisent des réseaux qui se caractérisent par l'imbrication étroite entre des sources d'origines diverses. Cette mobilisation des ressources externes, des savoirfaire (en complément des ressources internes) se présente comme un facteur critique du succès de l'innovation non seulement technologique, mais également commerciale (Gemünden et al., 1992). Contrairement aux grandes entreprises qui favorisent largement le développement des activités de R-D, l'introduction de l'innovation dans les PME semble davantage liée à l'acquisition ou à la création d'information de nature technique. Cependant, derrière cette homogénéité comportementale des PME se cache en réalité une diversité sectorielle qu'il convient d'analyser plus précisément.

\section{Combinaison des sources d'innovation technologique et structuration en réseaux: une analyse sous-sectorielle}

Bien qu'il puisse être fait état globalement d'une spécificité des PME en ce qui concerne leurs pratiques d'acquisition de ressources technologiques, l'hypothèse de la dépendance de la taille de l'entreprise par rapport à son appartenance sectorielle (Freeman, 1982) laisse supposer l'existence de divergences sectorielles quant à l'origine des sources d'innovation et à la structuration hétérogène des réseaux d'innovation. Pour vérifier cette hypothèse, nous avons isolé l'ensemble des sous-secteurs (en Nomenclature des activités produits [NAP] de niveau 600) de la base de données régionalisées de l'enquête innovation ${ }^{13}$. Ces sous-secteurs sont composés majoritairement de PME qui se sont déclarées innovantes. Ils comprennent au moins quinze entreprises (soit un total de 1592 entreprises réparties en 46 sous-secteurs listés en annexe). Ces entreprises relèvent de secteurs aussi divers que ceux

13. L'analyse que nous développons dans cette section, contrairement à la précédente, s'appuie donc sur les secteurs et non des données individuelles d'entreprises. 
des industries traditionnelles (textile, cuir, fonderie, papier et carton, travail mécanique du bois, ameublement, imprimerie), des biens d'équipements (fabrication de machines-outils, de matériel de manutention, d'équipements industriels, d'instruments et matériels de précision), des industries technologiquement intensives (parachimie, fabrication de matériels électriques, électroniques, de la construction de véhicules automobiles). Nombre des secteurs ainsi identifiés participent à la spécificité innovatrice de la région Rhône-Alpes, relativement à l'ensemble de la France (Guedj, 1993; Le Bas et Picard, 1994a). L'analyse sectorielle développée ici à un niveau relativement fin laisse apparaître des divergences quant à la répartition des sources d'innovation, d'une part, et à la structure du réseau d'acquisition des connaissances technologiques, d'autre part; divergences dont nous allons rendre compte.

\subsection{Spécificité sectorielle des sources d'innovation technologique}

La classification des secteurs notamment selon l'origine de leur innovation et le lieu où s'effectuent les dépenses de R-D a été initialement proposée par Pavitt (1984). Ce dernier distingue quatre grands types de secteurs: les secteurs dominés par les offreurs, ceux à rendements d'échelle croissants, les secteurs à offreurs spécialisés, les secteurs basés sur la science. On considère donc qu'il existe une logique sectorielle orientant la relation des entreprises non seulement à l'innovation, mais à l'origine de celle-ci. Nous analyserons les sources d'innovation déclarées par sous-secteurs suivant deux grandes perspectives. La première concerne la source principale de l'innovation, la seconde esquisse une dynamique combinatoire des sources mises en œuvre par chaque sous-secteur.

\subsubsection{Sources motrices de l'innovation dans les PME}

L'étude de la genèse de l'innovation nous a conduites à identifier trois sources critiques parmi les huits sources initialement répertoriées (à savoir l'activité d'étude technique, la R-D interne, l'acquisition d'intrants technologiques). Il s'avère que la source dominante d'introduction de l'innovation des PME n'est pas systématiquement l'activité d'étude technique et de méthode. Contrairement à ce qui apparaissait lors de l'analyse des PME, indépendamment de leur activité principale, on remarque que dans certains sous-secteurs l'innovation est mue principalement par l'utilisation de biens d'investissements ou par la mise en œuvre d'une activité de R-D interne. En effet, si $43 \%$ des sous-secteurs déclarent le plus fréquemment l'activité 
d'étude technique et de méthode comme source d'innovation, on dénombre autant de sous-secteurs citant l'utilisation de biens d'investissements comme source dominante. Ce résultat conforte l'idée que la plupart des PME innovent essentiellement à partir de ressources techniques (elles utilisent également d'autres sources, mais dans des proportions moindres) et que la diffusion de connaissances technologiques incorporées dans des biens d'investissement est un vecteur fondamental d'innovation pour les PME.

Parallèlement, près de $9 \%$ des individus déclarent la R-D interne comme source première d'innovation. Ils se nourrissent donc probablement plus d'informations scientifiques (sans pour autant négliger les autres sources) que ceux relevant des deux autres groupes précédemment singularisés ${ }^{14}$. Leur comportement en matière de R-D interne est incontestablement plus proche de celui qui caractérise les grandes entreprises que de celui qu'adoptent généralement les PME. Au demeurant, le pourcentage de soussecteurs utilisant à titre principal la R-D interne reste faible relativement à ceux qui utilisent l'activité d'étude technique et de méthode. L'une des hypothèses explicatives que l'on peut avancer pour justifier cette situation réside dans l'existence d'un biais statistique lié à la définition de l'activité de recherche et à la distinction établie entre R-D permanente et non permanente. En réalité, peu de PME déclarent faire de la recherche au sens normatif et réglementaire du terme, à savoir financer une activité de R-D organisée et permanente, mobilisant au moins un emploi de chercheur scientifique en équivalent temps plein. Mais cela ne signifie pas que toute recherche soit exempte de leur activité. Les disponibilités financières des PME rendent difficile le maintien en permanence de ce type d'activité. Aussi, disposent-elles d'une organisation de recherche plus souple, mise en place en quelque sorte à la demande, associée le plus souvent à l'activité d'études techniques qui intègre ainsi une activité de recherche non permanente.

\subsubsection{Combinaison des sources de l'innovation dans les PME}

Certaines sources d'innovation ne sont réellement efficaces que lorsqu'elles entrent en combinaison avec d'autres, tandis que d'autres vecteurs d'innovation sont plus autonomes. Ainsi, chacun des 46 sous-secteurs étudiés peut se différencier en fonction des combinaisons qu'il opère entre les différents vecteurs de l'innovation. L'idée sous-jacente est qu'il existe des sources d'innovation dont l'utilisation se suffit à elle-même et à partir desquelles

14. Il s'agit des sous-secteurs suivants : peintures, vernis et encres (1807), machines textiles (2410), équipement d'automatisation de processus industriels (2815), lunettes pour correction et protection de la vue (3403). 
l'obtention d'un effet apparaît comme relativement indépendant des autres sources. C'est le cas pour l'utilisation des biens d'investissements ou des matériaux nouveaux, qui favorisent la diffusion intersectorielle de l'innovation dans un laps de temps relativement réduit. Par contre, le dépôt de brevet ou l'acquisition de droits et licences... supposent qu'une activité de recherche, de développement ou d'étude ait été menée ${ }^{15}$ antérieurement ou simultanément. De façon plus générale, l'acquisition des sources externes implique la mobilisation de compétences internes (infra), esquissant une certaine dépendance de l'accès aux informations externes par rapport aux informations et connaissances spécifiques à la firme.

Nous avons eu l'occasion de souligner que les grandes entreprises déclaraient systématiquement un nombre plus élevé de sources que les PME. Or, on trouve également quelques secteurs caractérisés par un fort dynamisme en matière de combinaison des sources d'innovation, tandis que d'autres, plus nombreux, tendent à se focaliser sur quelques sources seulement. En effet, rares sont les secteurs qui déclarent sept sources ${ }^{16}$. En revanche, relativement nombreux sont ceux qui tendent à s'appuyer sur un nombre réduit de sources et qui présentent donc une faible diversification de leur pratique de création et acquisition des ressources innovatives ${ }^{17}$. Il s'agit, par exemple, de la construction métallique, de la chaudronnerie, de l'imprimerie de labeur, de l'emballage en matières plastiques, etc. Entre ces situations extrêmes, on trouve des secteurs tels que la fonderie des métaux non ferreux, les appareils électriques et électroniques..., le matériel médico-chirurgical et la robinetterie, lesquels secteurs manifestent une dynamique de diversification de leurs sources d'innovation technologique relativement active : combinant une multiplicité de sources, ils déclarent utiliser la plupart de celles-ci dans des proportions supérieures à la moyenne. Cette diversité peut s'expliquer au

15. Certes, des entreprises sont parfois construites par des entrepreneurs individuels sur la base d'une invention (brevet) qui leur est propre, mais il est peu probable que des entreprises de ce type soient dans notre échantillon, étant donné que le questionnaire concerne l'étude de l'activité d'innovation au cours des cinq dernières années effectuée par des entreprises de plus de vingt salariés (la probabilité est faible pour qu'en 5 ans, une entreprise se soit créée et ait dépassé le cap des 20 salariés).

16. Parmi l'ensemble des secteurs, six ont déclaré sept sources d'innovation. Les sources non citées sont la recherche externe pour le secteur 2115 , les brevets appartenant à l'entreprise pour les secteurs 2106, 4422, 5404 et l'acquisition de droits et de licences d'industrialisation pour les secteurs 4421 et 5111 .

17. Les secteurs peu diversifiés sont les suivants : 2106, 2108, 2408, 5110, 5111 , 5302,5404 . L'ensemble des sources est cité à un pourcentage relativement faible. 
niveau du secteur par des comportements différents selon les firmes (chacune privilégiant plutôt tel ou tel type de pratiques). Pour ces dernières, la diversité des pratiques peut résulter soit de la complexité de l'innovation à produire qui requiert des connaissances technologiques très diverses et qu'une firme seule ne peut par conséquent détenir, soit de la réalisation de plusieurs innovations au cours des cinq dernières années, innovations ayant chacune une origine différente.

L'analyse sous-sectorielle de l'origine principale et de la combinaison des sources de l'innovation met donc en relief l'absence d'unicité comportementale des PME. Cette disparité se retrouve également dans le développement des stratégies de réseaux. Nous avons évoqué les divers types de réseaux technoscientifiques qu'il était possible de distinguer théoriquement. L'existence d'un lien avec des acteurs extérieurs est appréhendée ici à partir de deux critères ${ }^{18}$ : la R-D externe acquise auprès d'organismes publics, professionnels, privés de recherche, de clients... et l'acquisition de droits et licences d'industrialisation relatifs à des brevets, inventions et savoir-faire techniques.

\subsection{Réseaux d'acquisition des connaissances technologiques externes à l'entreprise}

Globalement, $44 \%$ des PME déclarent utiliser la R-D externe comme source d'innovation (62\% pour les grandes entreprises), tandis qu'elles ne sont que $18 \%$ à acquérir des droits et licences pour innover (43\% des grandes entreprises). L'analyse sectorielle des pratiques d'innovation au niveau de la NAP 600 révèle des divergences dans les degrés d'immersion des PME dans des réseaux technologiques. Ainsi, parmi les 46 sous-secteurs étudiés, 31 sont fortement insérés dans des réseaux technologiques (soit plus de $67 \%$ ), c'est-à-dire qu'ils déclarent recourir plus que la moyenne à une source d'information externe (en l'occurrence la R-D externe acquise auprès d'organismes publics, professionnels, privés de recherche, de clients... ou par l'acquisition de droits et de licences d'industrialisation relatif à un brevet, une invention, un savoir-faire technique).

Précisons que nous optons ici pour une acception extensive de la notion de réseau. Celui-ci est compris comme un ensemble de relations entre des agents, des techniques. Notre hypothèse est que l'on peut parler de réseau

18. Le critère de R-D interne au groupe ne sera pas pris en considération compte tenu de son ambivalence. En effet, non seulement il est difficile d'affirmer systématiquement le caractère interne ou externe de cette source, mais un biais demeure du fait de notre méconnaissance du degré de dépendance des PME par rapport au groupe. 
technologique dès qu'une relation de nature technologique s'établit entre deux ou plusieurs acteurs, quand bien même l'objet de cette relation serait simplement l'acquisition d'informations (et non leur co-création). Une acception plus restrictive de la notion de réseau consisterait à identifier celui-ci non par l'existence d'une relation avec une autre entité, mais par l'affirmation d'une interrelation ou d'une interaction supposant une réciprocité systématique, stable, intense. Cette distinction sémantique n'est pas neutre au regard de la pertinence des indicateurs de réseau (Le Bas et Picard, 1994b). La conception la plus stricte du réseau suppose en effet de ne prendre en compte que les critères qui symbolisent un échange réel, une collaboration entre des agents qui participent en commun à la définition de l'objet qui les réunit. En revanche, l'optique définitionnelle extensive qui sera retenue ici autorise l'identification d'une structure en réseau dès qu'il y a un achat de connaissances technologiques externes liant au moins deux organismes. Cette hypothèse forte nous permet de considérer les éléments acquisition de droits et de licences d'industrialisation... et acquisition de recherche externe, comme des indices d'appartenance à un réseau. Dans cette perspective, trois grands groupes de secteurs seront identifiés : ceux dont les modalités d'accès aux ressources extérieures se déclinent sur un seul mode (R-D externe ou achat de droits) et ceux qui combinent ces deux sources ${ }^{19}$.

La relation d'une entreprise avec l'extérieur peut être objectivée par l'appel quasi exclusif à une activité de R-D externe. Une telle stratégie est développée par $15 \%$ des secteurs étudiés ${ }^{20}$, tels que ceux du décolletage, de la fabrication de machines-outils à métaux, de l'outillage, de produits de teintures, de chandails, de carrosseries et d'ouvrages tissés de l'industrie de la soierie (graphique 1, cadran 2). Compte tenu de la nature de ces secteurs, on peut considérer que la R-D externe est acquise essentiellement auprès d'organismes professionnels de recherche (tels que les Centres techniques) ou auprès de clients (notamment pour les secteurs appartenant à un réseau de sous-traitance comme le secteur carrosserie). La R-D externe acquise est essentiellement technique, appliquée pour les sous-secteurs appartenant à l'industrie textile ou au décolletage (sous-secteurs pour lesquels la R-D

19. Le pourcentage moyen d'entreprise par sous-secteurs déclarant utiliser la R-D externe comme source d'innovation est de $43 \%$ et de $18 \%$ pour l'achat de droits et de licences. Dans le graphique 1, l'intersection des axes est établie à ces valeurs moyennes.

20. Ces secteurs sont : $2104,2301,2303,3115,4418,4421,4432$. Le pourcentage d'entreprises déclarant utiliser cette source est supérieur à $45 \%$ (ce chiffre étant le pourcentage moyen déclaré de cette source pour l'ensemble de notre échantillon). Ces secteurs déclarent donc utiliser cette source dans une proportion supérieure à la moyenne. 


\section{GRAPHIQUE 1}

\section{Les réseaux technoscientiques des PME en Rhônes-Alpes}

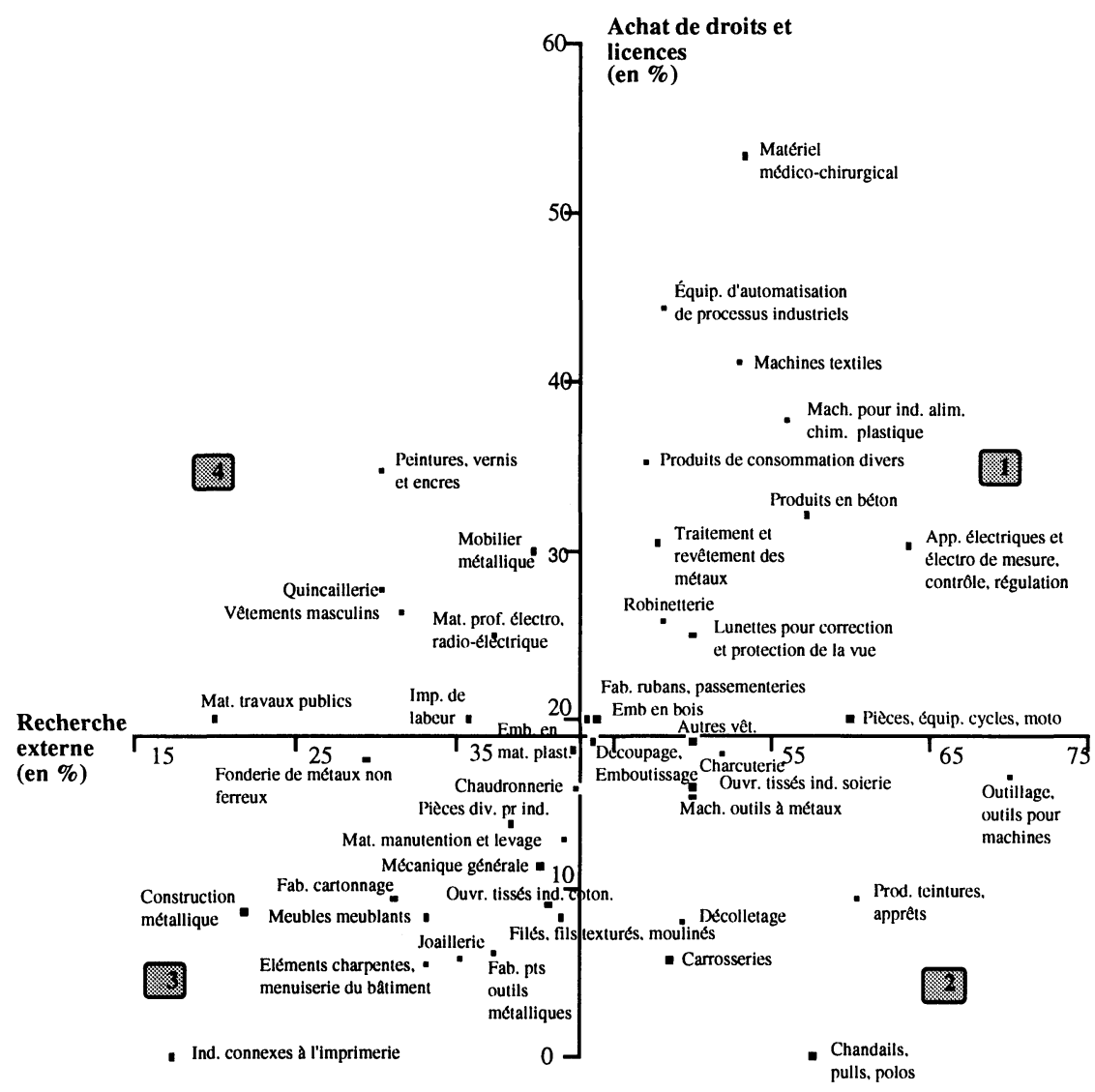

Source : SESSI (1990) et INSEE Rhône-Alpes (1993).

interne est une source d'innovation relativement peu déclarée). Elle traduit certainement l'existence d'un réseau d'acquisition de ressources de nature plus technique que scientifique.

Dans un second groupe, les secteurs développent une stratégie d'acquisition de ressources technologiques axée sur un autre vecteur d'information externe : l'achat de droits et licences d'industrialisation... Ce comportement 
caractérise $24 \%$ des secteurs (graphique 1, cadran 4). Pour deux tiers de ceux-ci, le brevet déposé ou acheté (sous forme de licence) est un vecteur non négligeable d'innovation. Il en est ainsi pour les sous-secteurs de fabrication de peintures..., d'emballages en matières plastiques, de la quincaillerie, du mobilier métallique, de la fabrication de matériels pour travaux publics qui déclarent également l'utilisation de brevets dont l'entreprise est titulaire comme une source importante d'innovation. Le tiers des sous-secteurs restant est composé d'entreprises déclarant recourir plus fréquemment à l'achat de droits et de licences d'industrialisation qu'à l'utilisation des brevets dont elles seraient titulaires. C'est le cas dans l'imprimerie de labeur, la fabrication de matériel professionnel électronique et radio-électrique, l'emballage en bois. L'achat de droit d'industrialisation n'est donc a priori pas lié systématiquement à la politique de dépôt de brevets des entreprises. En revanche, il se présente comme une source relativement dépendante des activités de recherche interne et d'étude technique, dans la mesure où pour sept des onze sous-secteurs concernés, ces activités sont une source dominante d'innovation.

Enfin, un troisième groupe rassemble les sous-secteurs qui mettent en œuvre des pratiques d'acquisition de connaissances extérieures plus complexes, recourant de façon relativement intense (par rapport à la moyenne) et simultanée aux deux sources d'informations externes identifiées (graphique 1 , cadran 1). Parmi les réseaux d'acquisition de ressources technologiques que nous avons identifiées, cette dernière est la plus développée, puisqu'elle concerne $28 \%$ des sous-secteurs, à savoir la fabrication de produits en béton, le traitement et revêtement des métaux, la robinetterie, la fabrication de machines pour industries alimentaires, chimiques et plastiques ou des machines textiles, les équipements d'automatisation de processus industriels, d'appareils électriques et électroniques de mesure... pour l'automatisme industriel, de pièces pour cycles, de lunettes pour correction et protection de la vue, de matériels médico-chirurgical, de la charcuterie, des autres vêtements de dessus de bonneterie et le secteur des produits de consommation divers. On retrouve parmi ces sous-secteurs ceux dont l'activité innovatrice est dominée par la recherche interne (à l'exception du secteur 1807, qui recourt moins à la R-D externe) ou par une activité d'étude, ce qui tend à confirmer l'existence d'une relation positive entre l'insertion des firmes et des sous-secteurs dans les réseaux d'acquisition technologique et leurs capacités propres à développer des activités de recherche et d'étude technique. En outre, on remarque que les sous-secteurs identifiés comme étant faiblement insérés dans des réseaux (graphique 1, cadran 3) sont également des sous-secteurs déclarant recourir peu à l'activité de R-D interne. 
Cette description cartographique des pratiques d'innovation peut être approfondie par une analyse des corrélations entre les différentes sources. Or, il apparaît qu'il n'existe pratiquement pas de corrélations entre les sources d'innovation ${ }^{21}$. Si l'on regarde ce qui se passe entre les deux sources externes d'innovation, on constate qu'il n'existe pas de corrélation pour l'ensemble des sous-secteurs. En effet, chacun des quatre groupes de sous-secteurs (identifiés dans le graphique 1) peut se résumer par un comportement relativement différent des autres pour ce qui concerne la relation entre les deux sources externes d'innovation. Nous avons également testé l'hypothèse d'une corrélation positive entre le recours à une activité de R-D externe et l'existence d'une activité de R-D interne. Une légère corrélation positive $(0,37)$ apparaît, indiquant une tendance pour la majorité des individus à déclarer recourir d'autant plus à la R-D externe qu'ils affirment effectuer de la R-D interne. Ainsi, l'existence d'une relation entre la capacité de recherche d'une firme et sa capacité de rechercher, de capter des informations externes n'est pas infirmée (Cohen et Levinthal, 1989).

En définitive, s'il a été possible d'identifier un comportement de création et d'acquisition de ressources technoscientifiques globalement homogène pour les PME de la région Rhône-Alpes, il est frappant de constater à quel point les comportements sont finalement différents pour ces mêmes PME lorsqu'on les appréhendent suivant une approche sous-sectorielle. Des différences apparaissent non seulement au niveau de la principale source d'innovation déclarée ou de la dynamique combinatoire de ces sources, mais également au niveau de la construction des relations de réseau en vue de l'acquisition d'informations ou de connaissances technologiques externes. En outre, on s'aperçoit que des sous-secteurs identifiés au niveau de la NAP 600 qui relèvent d'un même intitulé sectoriel en NAP 100 présentent également des comportements très hétérogènes eu égard aux sources de leur innovation technologique (cela est particulièrement frappant dans le cas du secteur textile) $)^{22}$.

21. Une analyse en composantes principales réalisée sur l'ensemble des secteurs et indépendamment de la taille de l'entreprise indique que d'une façon générale, les secteurs d'activité qui innovent beaucoup, innovent indifféremment de la source utilisée, de même que ceux qui sont faiblement innovant le sont également quelle que soit la source. Ce résultat confirme l'existence d'une complémentarité entre les différentes sources de l'innovation.

22. L'explication de ces disparités, au-delà de leur simple description, nécessiterait une étude beaucoup plus détaillée qui renverrait en définitive à la mise en évidence du caractère «firme ayant une stratégie de différenciation » de la construction des compétences et connaissances nécessaires à l'innovation. 


\section{Conclusion}

L'analyse des sources de l'innovation technologique à partir de l'enquête régionalisée du SESSI a permis de montrer le caractère très diversifié des pratiques innovatrices des PME. Celui-ci s'exprime sur un plan sectoriel, non seulement par la source principale de l'innovation et des modes de combinaison des différentes sources entre elles, mais également par l'immersion des entreprises dans les réseaux d'acquisition de connaissances technologiques externes. Ce résultat nous conduit à formuler deux remarques finales.

Cette description de l'hétérogénéité des pratiques innovatrices invite à une analyse des stratégies de réseaux des PME. Elle implique en définitive de considérer la construction des réseaux technologiques comme un phénomène complexe: il convient d'identifier précisément les différents acteurs avec lesquels les firmes entrent en contact, de cerner simultanément les réseaux d'acquisition et de création (en collaboration) d'innovations, pour ensuite analyser l'importance relative de la contribution et la hiérarchisation des différentes sources (internes et externes) à la performance innovatrice et économique des entreprises. Des études spécifiquement construites sur une problématique de réseau d'innovation visent à expliquer la logique sousjacente au déploiement de stratégies de réseaux, à leur territorialité (Héraud et Nanopoulos, 1994), à leur impact sur les performances innovatrices des firmes (Gemünden et al., 1992), à la hiérarchisation des sources d'innovation technologique.

Par ailleurs, ces éléments de la diversité des pratiques innovatrices des PME renvoient à la difficulté de la mise en œuvre d'une politique technologique trop globale ou axée trop exclusivement sur un type de source d'innovation. Il convient de prendre en compte non seulement chaque agent ou chaque source indépendamment les uns des autres, mais aussi les relations qu'ils entretiennent entre eux. Car c'est bien dans la capacité que possède chaque entreprise de rechercher puis de combiner entre elles des connaissances d'origines diverses que réside son potentiel innovateur. On tient probablement là une des raisons qui expliquerait la plus forte innovativité des entreprises de grande taille relativement aux PME. 


\section{AnNeXe \\ Nomenclature Activités Produits (niveau 600)}

\begin{tabular}{|c|c|}
\hline Code & Intitulé du secteur \\
\hline 1508 & Produits en béton \\
\hline 1807 & Peintures, vernis et encres \\
\hline 2002 & Fonderies de métaux non ferreux \\
\hline 2102 & Découpage, emboutissage \\
\hline 2103 & Traitement et revêtement des métaux \\
\hline 2104 & Décolletage \\
\hline 2106 & Construction métallique \\
\hline 2108 & Mécanique générale \\
\hline 2111 & Quincaillerie \\
\hline 2113 & Mobilier métallique \\
\hline 2115 & Fabrication de petits outils métalliques \\
\hline 2301 & Machines outils à métaux \\
\hline 2303 & Outillage, outils pour machines \\
\hline 2401 & Robinetterie \\
\hline 2408 & Chaudronnerie \\
\hline 2409 & $\begin{array}{l}\text { Machines pour industries alimentaires, } \\
\text { chimiques et plastiques }\end{array}$ \\
\hline 2410 & Machines textiles \\
\hline 2501 & Matériels de travaux publics \\
\hline 2503 & Matériels de manutention et de levage \\
\hline 2815 & $\begin{array}{l}\text { Équipements d'automatisation de } \\
\text { processus industriels }\end{array}$ \\
\hline 2913 & $\begin{array}{l}\text { Appareils électriques et électroniques } \\
\text { de mesure, appareils de contrôle et de } \\
\text { régulation pour l'automatisme industriel }\end{array}$ \\
\hline 2914 & $\begin{array}{l}\text { Matériel professionnel électronique et } \\
\text { radio-électrique }\end{array}$ \\
\hline 3115 & Carrosseries, bennes, remorques \\
\hline
\end{tabular}

\begin{tabular}{|c|c|}
\hline Code & Intitulé du secteur \\
\hline 3117 & $\begin{array}{l}\text { Pièces et équipements pour cycles et } \\
\text { motocycles }\end{array}$ \\
\hline 3403 & $\begin{array}{l}\text { Lunettes pour correction et protection de la } \\
\text { vue }\end{array}$ \\
\hline 3406 & Matériel médico-chirurgical et prothèses \\
\hline 3504 & Charcuteries et conserves de viandes \\
\hline 4417 & $\begin{array}{l}\text { Filés et fils texturés et moulinés de soie, } \\
\text { textiles artificiels et synthétiques }\end{array}$ \\
\hline 4418 & $\begin{array}{l}\text { Produits des teintures, apprêts et } \\
\text { impressions }\end{array}$ \\
\hline 4421 & Chandails, pulls, polos, gilets \\
\hline 4422 & Autres vêtements de dessus de bonneterie \\
\hline 4430 & $\begin{array}{l}\text { Ouvrages tissés des industries cotonnières } \\
\text { et linières }\end{array}$ \\
\hline 4432 & Ouvrages tissés de l'industrie de la soierie \\
\hline 4442 & $\begin{array}{l}\text { Fabrication de rubans, tresses, } \\
\text { passementeries }\end{array}$ \\
\hline 4701 & Vêtements masculins \\
\hline 4802 & $\begin{array}{l}\text { Éléments de charpentes et menuiseries du } \\
\text { bâtiment }\end{array}$ \\
\hline 4805 & Emballage en bois \\
\hline 4901 & Meubles meublants \\
\hline 5007 & Fabrication cartonnage \\
\hline 5110 & Imprimerie de labeur \\
\hline 5111 & Industries connexes à l'imprimerie \\
\hline 5301 & $\begin{array}{l}\text { Mélanges, plaques, films, tubes, tuyaux et } \\
\text { profilés }\end{array}$ \\
\hline 5302 & Pièces diverses pour l'industrie \\
\hline 5303 & Emballages en matières plastiques \\
\hline 5305 & Produits de consommation divers \\
\hline 5404 & Bijouterie, joaillerie \\
\hline
\end{tabular}




\section{Bibliographie}

ACS, Z.J. et D.B. AUDRETSCH (1988), «Innovation in the large and small firms : an empirical analysis », American Economic Review, vol. 78, p. 678-690.

ACs, Z.J. et D.B. AUdRetsch (1990), Innovation and Small Firms, Cambridge, Mass., MIT Press.

AUZEBY, F. et J.P. FRANÇOIS (1992), «L'Innovation technologique dans l'industrie», Le 4 Pages, SESSI.

BARRÉ, R. (1991), "Quelques résultats préliminaires de l'enquête innovation », $L a$ Lettre $O S T, \mathrm{n}^{\circ} 3$, avril.

BARRY (de), C. et B. SAVOYE (1993), «Les petites entreprises innovantes », Le 4 pages, INSEE, $\mathrm{n}^{\circ} 268$, juillet.

Bernard, J. et A. Torre (1994), «Les dynamiques d'innovation et de R-D dans les PMI françaises », Revue Internationale PME, vol. 7, $\mathrm{n}^{\text {os }}$ 3-4.

Bidault, F. (1993), «Apprentissage et réseaux », Économies et Sociétés, Série Dynamique technologique et organisation, $W, n^{\text {os }} 1-5$, p. 79-101.

Callon, M. (1991), "Réseaux technico-économiques et irréversibilités », dans R. Boyer et al. (dir.), Les Formes de l'irréversibilité en économie, Paris, EHESS.

CoHEN, W.M. et D. LEVINTHAL (1989), «Innovation and learning: the two faces of R-D », Economic Journal, vol. 99, septembre, p. 569-596.

COMMISSARIAT GÉNÉRAL DU PLAN (Rapport), (1993), Recherche et innovation: le temps des réseaux, Paris, La Documentation française.

DE BRESSON, C. et F. AMESSE (1991), « Networks of innovators : a review and introduction to the issue », Research Policy, vol. 20, n 5, p. 363-380.

DodGSON, M. (1991), «Technology learning, technology strategies and competitive pressures », British Journal of Management, vol. 2, p. 133-149.

Dosi, G. (1988), «Sources, procedures and microeconomic effects of innovation », Journal of Economic Literature, vol. 26, $\mathrm{n}^{\circ} 3$, p. 1120-1171.

Dussauge, P. et B. Ramanantsoa (1987), Technologie et stratégie d'entreprise, Paris, McGraw-Hill.

FrançOIS, J.P. (1991), «Une Enquête sur l'innovation », Courrier des statistiques, $\mathrm{n}^{\circ}$ 57, mars, p. 11-15.

FRASCATI (de), M. (1981), La Mesure des activités scientifiques et techniques, OCDE.

FREEMAN, C. (1982), The Economics of Industrial Innovation, Londres, Frances Pinter.

Gemünden, H.B., H. Heydebreck et R. HERden (1992), «Technological interweavement: a means of achieving innovation success », $R$-D Management, vol. $22, n^{\circ} 4$.

GRANSTRAND, O. et S. SJÖLANDER (1990), «The acquisition of technology and small firms by large firms », Journal of Economic Behavior and Organization, vol. 13, p. 367-386. 
GuEdJ, B. (1993), «L'Innovation en Rhône-Alpes », Rapport de stage DESS EMIT Lyon 2, INSEE Rhône-Alpes, septembre.

HÉrAud, J.A. et K. NANOPOUlos (1994), «Les réseaux de l'innovation dans les PME : illustration sur le cas de l'Alsace », Revue Internationale PME, vol. 7, nos 3-4.

JAFRA, C. et L. ZINS (1993), «La Lorraine dans la course», Économie Lorraine, $\mathrm{n}^{\circ} 117$, janvier.

Kelley, M.R. et H. BrooKS (1991), «External learning opportunities and the diffusion of process innovations to small firms », Technological Forecasting and Social Change, vol. 39, p. 103-125.

KLEINKNECHT, A. (1987), "Measuring R-D in small firms : how much are we missing ?», The Journal of Industrial Economics, vol. 36, $\mathrm{n}^{\circ} 2$, p. 253-256.

KLINE, S.J. (1985), «Innovation is not a linear process », Research Management, juillet-août, p. 36-45.

KLINE, S.J. et N. RosenberG (1986), «Innovation : an overview », dans R. Landau et N. Rosenberg (dir.), The Positive Sum Strategy, National Academy Press.

LE BAS, C. (1993), «La firme et la nature de l'apprentissage», Économies et Sociétés, Série Dynamique technologique et organisation, W, vol. 1, ${ }^{\circ}$ 5, p. 7-24.

LE BAS, C. et F. PICARD (1994a), «Système d'innovation régional : une analyse à travers les sources d'innovation technologique », Communication à la Journée d'Étude du CREUSET sur Les politiques technologiques régionales, 19 mai.

LE BAS, C. et F. PICARD (1994b), « Réseaux technologique et innovamétrie : l'apport de la statistique d'innovation à l'analyse des réseaux », Document de travail LESA.

MOMIGLIANI, F. et D. SinISCALCO (1984), «Technology and international specialization », Banca Nazionale del Lavoro Quarterly Review, ${ }^{\circ} 154$, p. 257-284.

MOWERY, D.C. (1983), «The relationship between intrafirms and contractual forms of industrial research », Explorations in Economic History, vol. 20, p. 351-374.

PAVITT, K. (1984), « Sectoral patterns of technical change : towards a taxonomy and a theory ", Research Policy, vol. 13, p. 343-373.

Powell, W.W. (1990), "Neither markets nor hierarchies: networks forms of organization », Research in Organizational Behavior, vol. 12, p. 295-336.

RoBSON, M., J. TOWNSEND et K. PAVITT (1988) «Sectoral patterns of production and use of innovations in the UK: 1945-1983», Research Policy, vol. 17, p. 1-14.

Rothwell, R. et W. Zegveld (1982), Innovation and the Small and Medium Sized Firm, Londres, Frances Pinter.

SCHERER, F.M. (1982), "Inter-industry technology flows in the United States », Research Policy, vol. 11, n 4, p. 227-245.

TERLECKYJ, N.E. (1974), Effects of R-D on the Productivity Growth Industries : An Explanatory Study, New York, National Planning Association.

Thorelli, H.B. (1986), « Networks : between markets and hierarchies », Strategic Management Journal, vol. 7, p. 37-51. 